SSCL --297

DE91 004999

\title{
SELECTION OF BASIC PARAMETERS FOR THE COLLIDER RINGS
}

\author{
D. A. Edwards and M. J. Syphers \\ Superconducting Super Collider Laboratory* \\ Accelerator Division \\ 2550 Beckleymeade Avenue \\ Dallas, Texas 75237
}

June 1990

\section{DISCLAIMER}

\begin{abstract}
This report was prepared as an account of work sponsored by an agency of the United States Government. Neither the United States Government nor any agency thereof, nor any of their employees, makes any warranty, express or implied, or assumes any legal liability or responsibility for the accuracy, completeness, or usefulness of any information, apparatus, product, or process disclosed, or represents that its use would not infringe privately owned rights. Refer. ence herein to any specific commercial product, process, or service by trade name, trademark, manufacturer, or otherwise does not necessarily constitute or imply its endorsement, recommendation, or favoring by the United States Government or any agency thereof. The views and opinions of authors expressed herein do not necessarily state or reflect those of the United States Government or any agency thereof.
\end{abstract}

*Operated vy the Universities Research Association, for the U.S. Department of Energy under Contract No. DE-AC02-89ER40486. 


\title{
Selection of Basic Parameters for the Collider Rings
}

\author{
D. A. Edwards and M. J. Syphers \\ SSC Laboratory, Dallas, TX
}

June 4, 1990

\section{Introduction}

This paper describes the choices of three major accelerator parameters for the Collider rings of the SSC. These are the quadrupole spacing in the arcs, the injection energy, and the coil diameter of the superconducting dipole magnets. These parameters have significant cost and performance impact and for these reasons have been under intensive review throughout the SSC design process. The main purpose of this paper is to document the reasons for recent recommended changes in these parameters.

Four years have elapset since the issuance of the Conceptual Design Report by the Central Design Group. The only major parameter change since then and before the formation of SSC Laboratory was the increase in quadrupole spacing from 96 meters to 114.25 meters associated with an increase of the phase advance per cell from 60 degrees tc 90 degrees. It should not be surprising that the selection of the site brought with it a reexamination of the design incorporating the work of the last four years.

The next section presents the motivations for the recommended changes. The accelerator physics issues related to the three parameters are discussed in the succeeding section. Since this material represents the work of many people, as reflected by the references in the text, the present authors are only attempting to summarize their work. Any errors of interpretation are our own.

\section{Motivations for Charge}

There have been many developments since the 1986 Conceptual Design Report was issued which have led to the recommendation for changes in some of the Collider paraineters. Accelerator experiments have been performed at Fermilab and at CERN which revealed long-term particle beam loss mechanisms in the presence of strong nonlinear guide fields. Computer simulations have shown that particles which seem to lie on stabie orbits when tracked for only a few hundred turns may actually be lost if allowed to circulate for many thousands 
of turns in the presence of strong nonlinearities. In addition, operational experience with the first superconductiny colliding beams accelerator, the Fermilab Tevacron, has uncovered a time dependence of the persistent current multipoles present in superronducting magnets which, though familiar in solid state physics, had not been anticipated by the accelerator community. These developments are described further in the subsections to follow.

\subsection{Experiments}

Three major studies of existing accelerators have been performed recently at Fermilab and CERN to study the transverse dynamics of particles in nonlinear systems. Two of these studies were performed on relatively "linear" machines (the Tevatron and the SpS) in which nonlinear magnetic fields of known strength were introduced. The third study was of the Fermliab Main Ring at its injection energy, an inherently nonlinear environment. An overview of these experiments can be found in [1].

Fermilab experiment E778 has developed into a collaborative effort in the study of nonlinear dynamics and beam diagnostics involving accelerator physicists from the SSC Laboratory, Cornell, SLAC, LBL, CERN, and Fermilab [2]. The experiment was organized in 1986 at the request of a HEPAP subcommittee to verify the magnet aperture criterion proposed for the SSC [3]. The criterion emphasized the need to have particle motion deviate as little as possible from linear behavior and was stated in the language of smear and tune-shift allowances. One aspect of the experiment has consisted of powering a distribution of sextupole magnets in the Tevatron and inducing betatron oscillations of the beam using a pulsed kicker magnet; the turn-by-turn beam position monitoring system of the Tevatron, modified for the experiment, could then collect many thousands of turns of position data which could then be analyzed. The measurements were able to confirm smear and tune-shift predictions from short-term tracking and analytical calculations. These results, plus the first direct measurements of particles trapped in resonance islands, would have satisfied the goals of the experiment.

However, a surprising result of the expcriment was the observation of a reduction of the beam lifetime associated with the sextupoles. (See Figure 1.) The lifetime could be improved by turning off the RF accelerating system and could be varied by modulating the tune with pulsed quadrupoles. This observation was one of the early warning signs leading to the question of long-term stability in the SSC at injection.

Meanwhile, similar beam studies were being conducted at CERN on the SpS at an energy of $120 \mathrm{GeV}$ where the bend magnet fields are known to be very linear. Again a collection of sextupoles were powered and beam measurements were performed. The initial studies concentrated on comparing the measured dynamic aperture of the machine in the presence of the sextupole fields with those obtained from computer simulations [4]. The measurements indicated a dynamic aperture smaller (by a factor of two) than that predicted by the initial computer simulations. Only when the computer simulations included more physics (such as closed orbit offsets through the sextupoles) could one account for the measured dynamic aperture. 


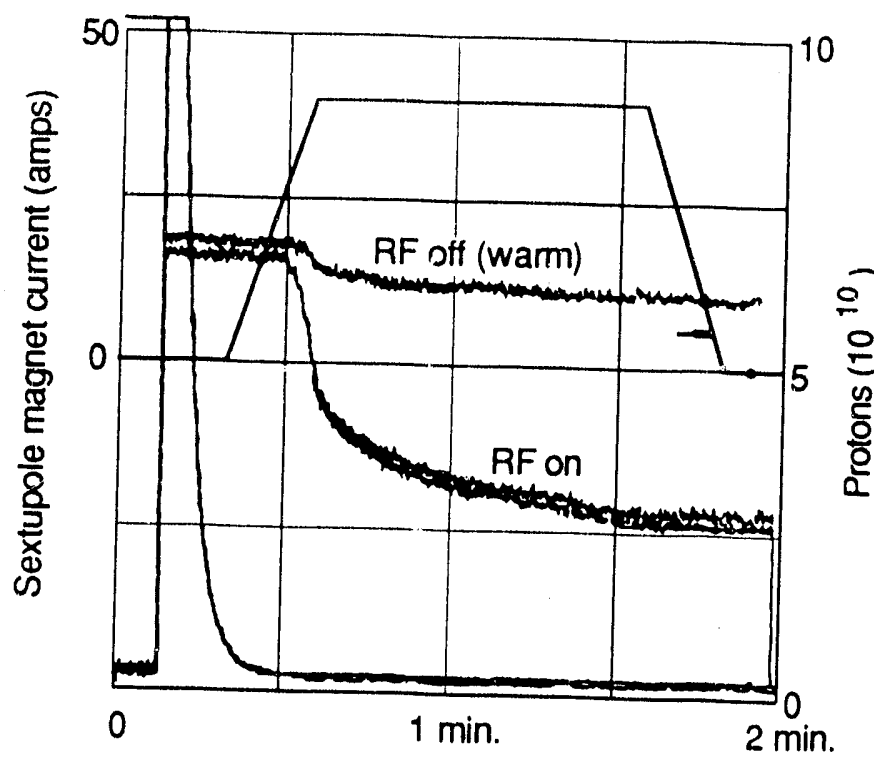

TIP-12222

Figure 1: Beam intensity as function of time in the Tevatron with and without the RF system enabled. The trapezoidal curve indicates the period during which the sextupoles
were powered.

As in the E778 experiment, a slow beam loss in the SpS could be observed at strong sextupole excitations and one of the experiments attempted to measure the rate of this diffusion [5]. The results suggested a significant diffusion rate at amplitudes corresponding to relatively small values of smear and tuneshift.

Stimulated by the CERN experiment, diffusion measurements were performed in the Tevatron during the June 1989 run of E778. Using a beam kicker and the flying wire profile monitoring system, the bearn distribution was measured as a function of time for various kick amplitudes. The width of the beam was observed to vary with time in the presence of the strong sextupoles. Preliminary results are shown in Figure 2. The flying wire system hardware is to be impreved and the measurements repeated during the next run of E778.

The causes and mechanisms of the observed diffusion remain to be studied. It should be emphasized that no diffusion was observed in either experiment when the sextupoles were not energized.

A third important set of accelerator studies have been taking place at Fermilab using the Main Ring synchrotron [6]. A set of dynamic aperture experiments on this machine began in 1987 when the laboratory was considering alternate injection energies. At the present injection energy of $8.9 \mathrm{GeV}$, the beam intensity lifetime is poor, on the order of $5 \mathrm{sec}$. or worse. Unlike the experimental situations found in the Tevatron and the SpS at $120 \mathrm{GeV}$, the Main Ring injection environment is such that the natural dynamic aperture is on the order of the emittance of the incoming beam from the Booster synchrotron; in many instances it is smaller. One of the more striking results of the Main Ring experiments is the time development of the beam enittance toward an equilibrium value. This development, is indicated in Figure 3. The horizontal and vertical emittances are plotted as functions of time for up to 60 sec. after injection. The large initial emittances (12-14 $\pi$ mm-mr; $95 \%$, 


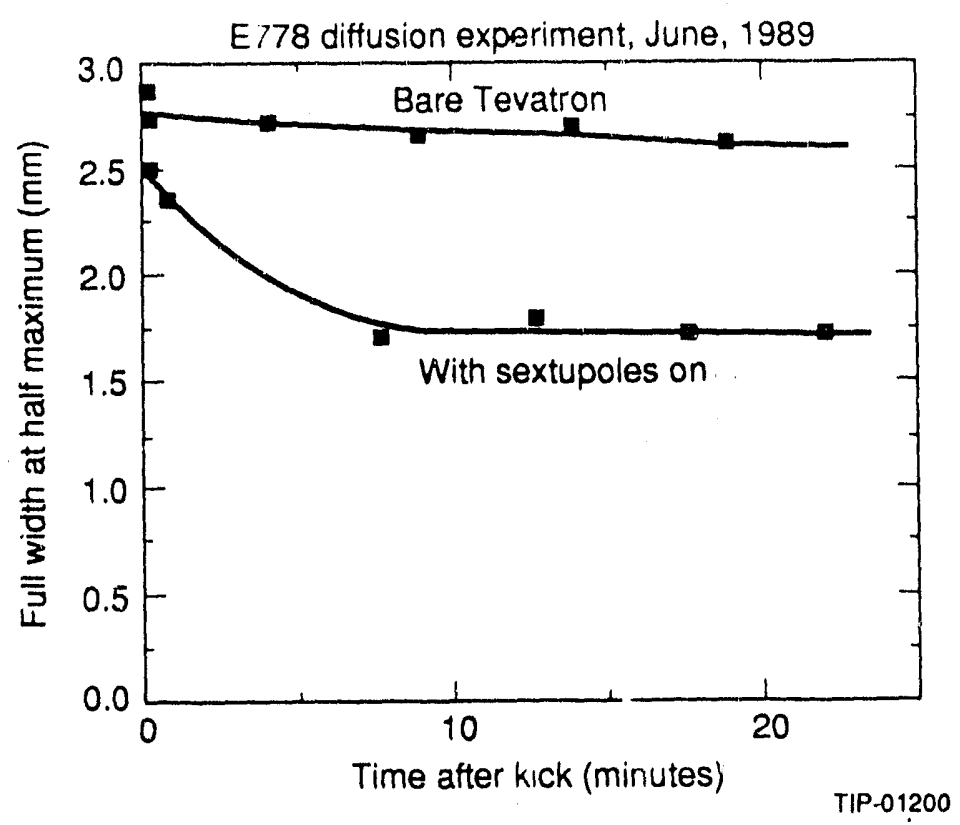

Figure 2: Diffusion measurements from E778. The beam width measured as a function of time is displayed for sextupoles on and for sextupoles off.

normalized) are indicative of high intensity beams from the Booster. The smaller emittances were obtained using beam scrapers located in the injection line. As can be seen, equilibrium emittances $\left(\epsilon_{x} \sim 7 \pi \mathrm{mm}-\mathrm{mr}, \epsilon_{y} \sim 5 \pi \mathrm{mm}-\mathrm{mr}\right)$ are obtained after about $25 \mathrm{sec}$. at $8.9 \mathrm{GeV}$. Correspondingly, the large incoming beams have a short lifetime which lengthens as time goes on, while the smaller incoming beams start out with a longer lifetime. In each case the same equilibrium lifetime eventually is reached. The measured physical aperture of the Main Ring would correspond to a phase space area more than three times the equilibrium emittances quoted above.

Initial Main Ring measurements of 1987 , at both $8.9 \mathrm{GeV}$ and $20 \mathrm{GeV}$, indicated that the emittance growth rates and, upon reaching an aperture, the equilibrium lifetimes were consistent with diffusion due to beam-gas scattering [7]. Later experiments, involving variation of vacuum pressure within the beam chamber, have supported this model. With this understanding, subsequent studies have concentrated on the source of the aperture.

In early experiments it was noted that turning off the RF system dramatically increased the intensity lifetime. Later experiments showed that the emittance growth due to RF noise is negligible and that it is indeed the dynamic aperture which increases when synchrotron oscillations are not present.

After 20 years of operation the beam behavior in the Main Ring is not understood. Though the Main Ring was not designed as a storage ring, experience with this machine prompts one to look even more closely at the particle dynamics associated with the SSC at its injection energy. 

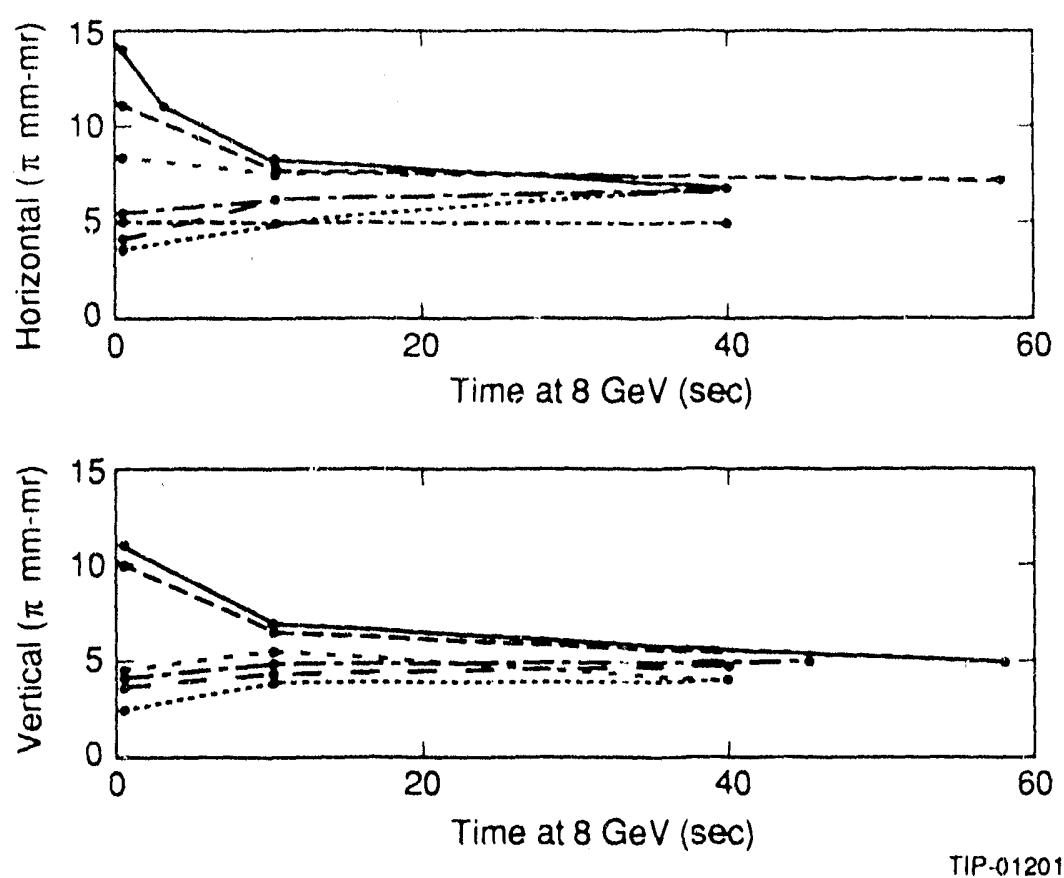

Figure 3: Main Ring emittance vs. time for beam with varying injection emittances.

\section{$2.2 \quad$ Tracking}

Computer simulations in which particles are tracked through the magnetic elements of a synchrotron were used to perform calculations of the linear aperture of the SSC for the 1986 CDR. Typically these calculations consisted of tracking particles for a few hundred up to a few thousand revolutions. However, during the filling sequence of the Collider rings, the first particles that are injected must successfully circulate for about 30 minutes ( 6 million turns) and so the tracking calculations needed to be extended several orders of magnitude.

To be able to predict the long-term behavior of particles, the model must incorporate significant features of the three degree-of-freedom problem. As mentioned earlier, experimental measurements of the $\mathrm{SpS}$ dynamic aperture in the presence of strong sextupoles yielded values a factor of two smaller than what had been predicted by tracking particles for only a small number of turns. To generate the observed dynamic aperture in the simulations synchrotron oscillations and closed orbit errors (on the order of those actually in the machine) through the sextupoles had to be incorporated into the model and particles had to be tracked for $10^{5}$ turns [8]. (See Figure 4.)

At Fermilab, extensive computational studies of the Main Ring at injection have been performed in order to understand the observed dynamic aperture and leam lifetime [9]. The model included multipole errors in the main dipole magnets up to 5 -th order. The distribution of errors was derived from measurements performed on a set of 30-40 dipole magnets. The 10and 12-pole coefficients of the multipoles were adjusted until the tracking produced the same variations of tune with momentum as those observed in the real machine. (The adjustments were small.) Particles were then tracked for up to 3.5,000 revolutions, corresponding to the time spent at the injection energy.

The Main Ring simulations showed particles that would appear to be stable for many thousands of turns, but then would abruptly leave the accelerator in a small number of 


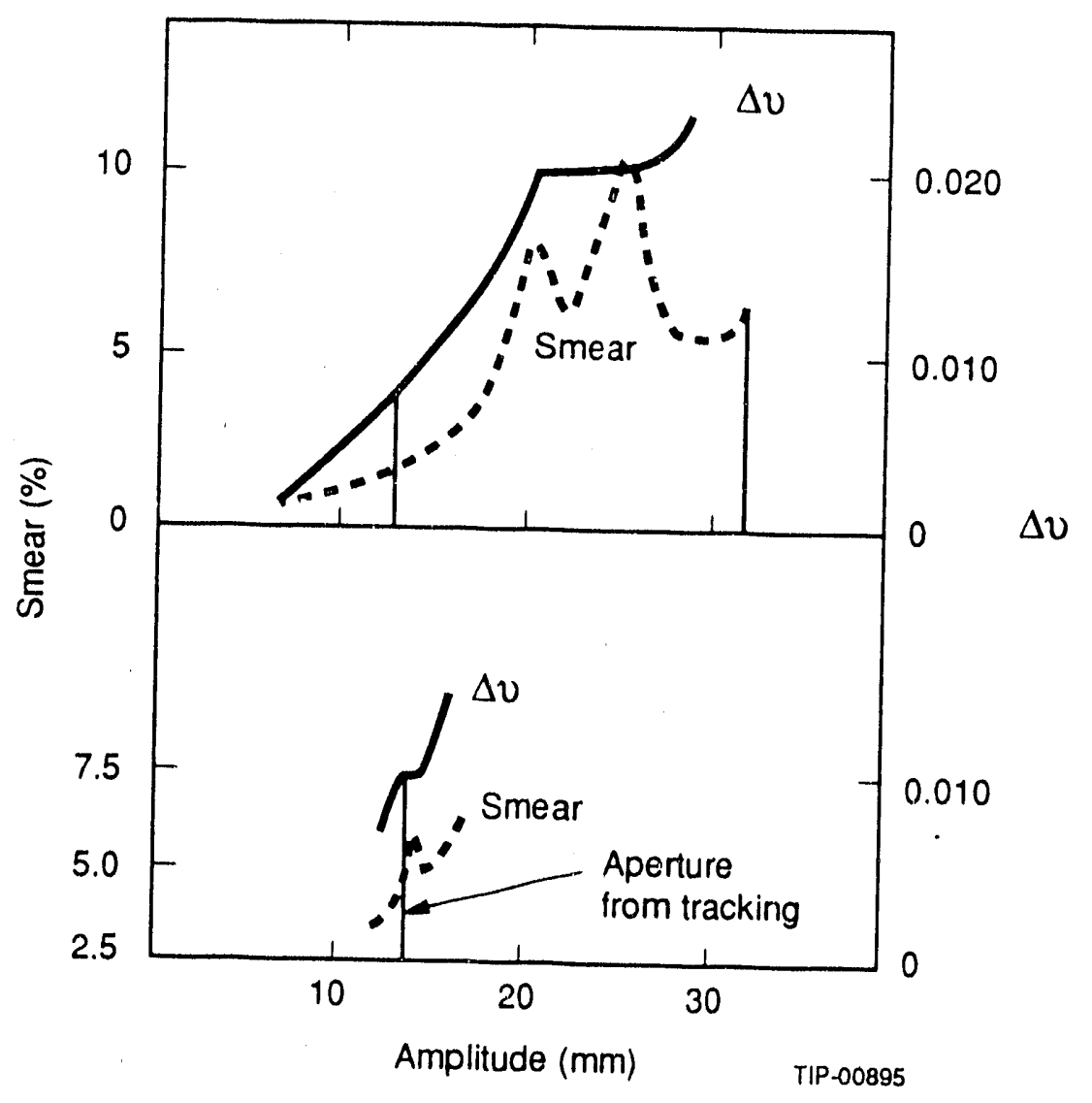

Figure 4: The top graph shows smear and tune shift as functions of amplitude determined from short-term tracking in the absence of orbit errors for the CERN experiment. The aperture (left). The botthe calculated dynamic aperture (right) and the measured dynamic orbit errors. 


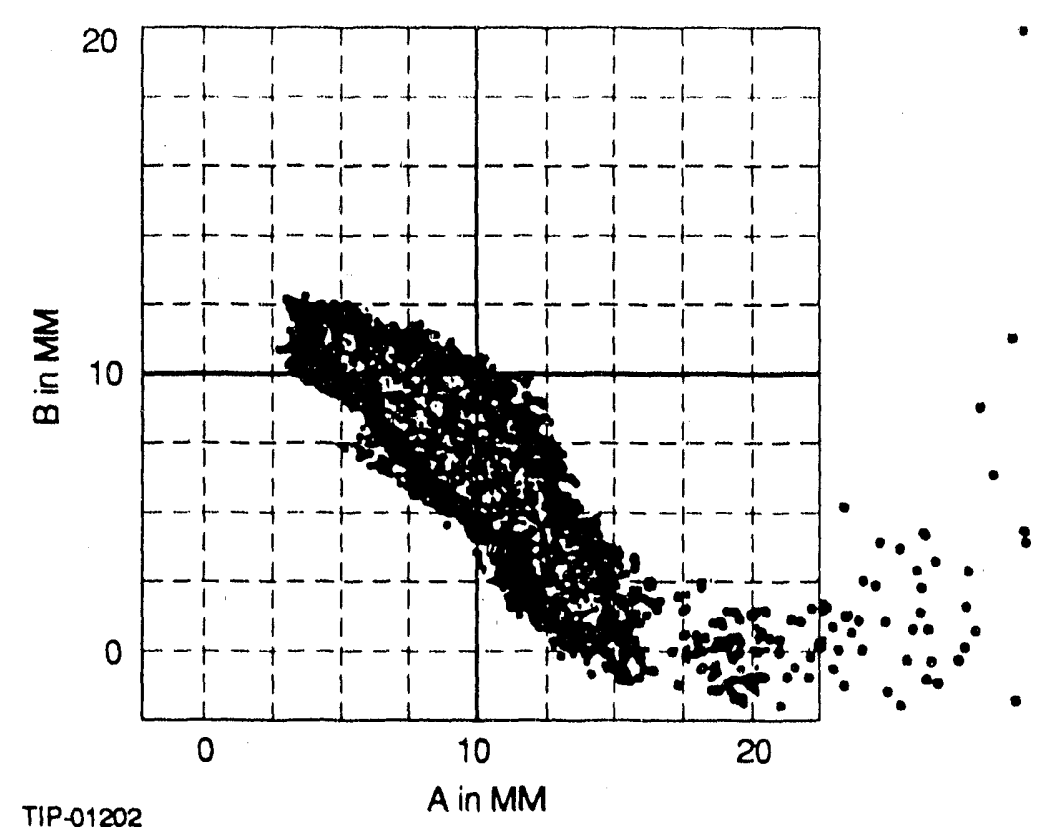

Figure 5: Vertical oscillation amplitude vs. horizontal oscillation amplitude for a particle tracking simulation of the Main Ring. The particle survived 33,000 turns before exhibiting rapid horizontal emittance growth.

revolutions. (See Figure 5.) The number of turns a particle survives is very sensitive to the particle's initial oscillation amplitude as indicated in Figure 6. While the survival time increases as the initial amplitude decreases, there is no clear cut-off. The amplitude at which particles survive the entire injection process corresponds roughly with the measured dynamic aperture. It should be noted that these results were obtained from tracking which included the detailed description of the magnets, synchrotron oscillations, and an accurate description of the closed orbit.

Luring the winter of 1988-89 studies of long-term stability were begun in which particles were tracked through a CDR Collider ring typically for as much as $10^{4}$ revolutions using a CRAY supercomputer [10]. Tracking through each element of a Collider ring for much more than about $10^{5}$ turns was considered prohibitively expensive and time consuming. When the results werı displayed as survival plots, as in the Main Ring simulations, again no clear dynamic apertide was visible. A surviva! plot for a simulation of the Collider as prepared according to the CDR parameters is shown in Figure 7. However, as will be discussed later, it is now considered essential to assure the commissioning and the smooth operation of the Collider under a more demanding environment than as envisioned in the CDR design. When this environment is simulated, the resulting survival plot is that of Figure 8 . If the data were simply extrapolated to the left, these results might suggest that the dynamic aperture for a particle to survive $10^{7}$ turns would be on the order of one millimeter. 'Though this might be a slight underestimate, nevertheless it is hard to feel comfortable with the long-term dynamic aperture of this machine. 


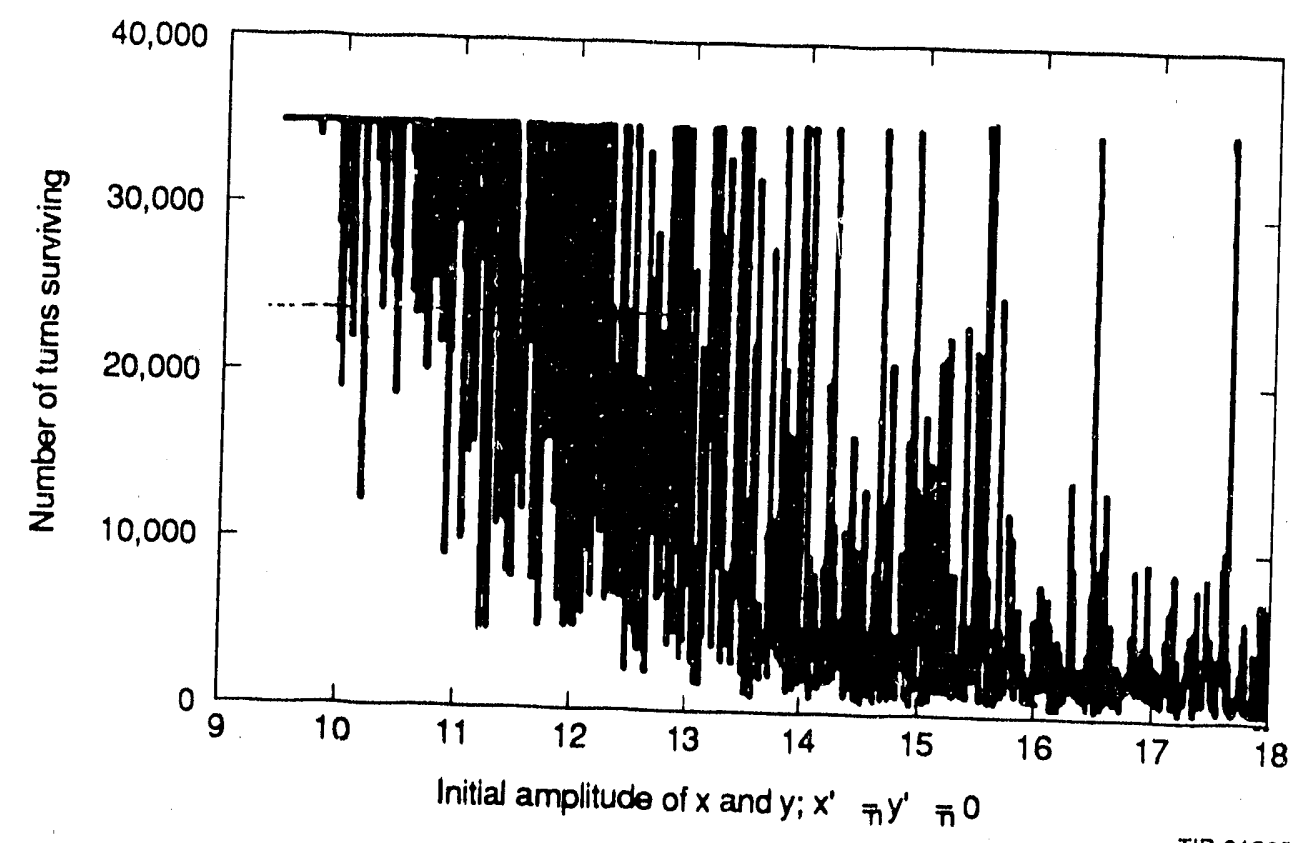

Figure 6: Number of turns survived in Main Ring simulation vs. initial betatron oscillation amplitude. The step size in amplitude was $0.01 \mathrm{~mm}$.

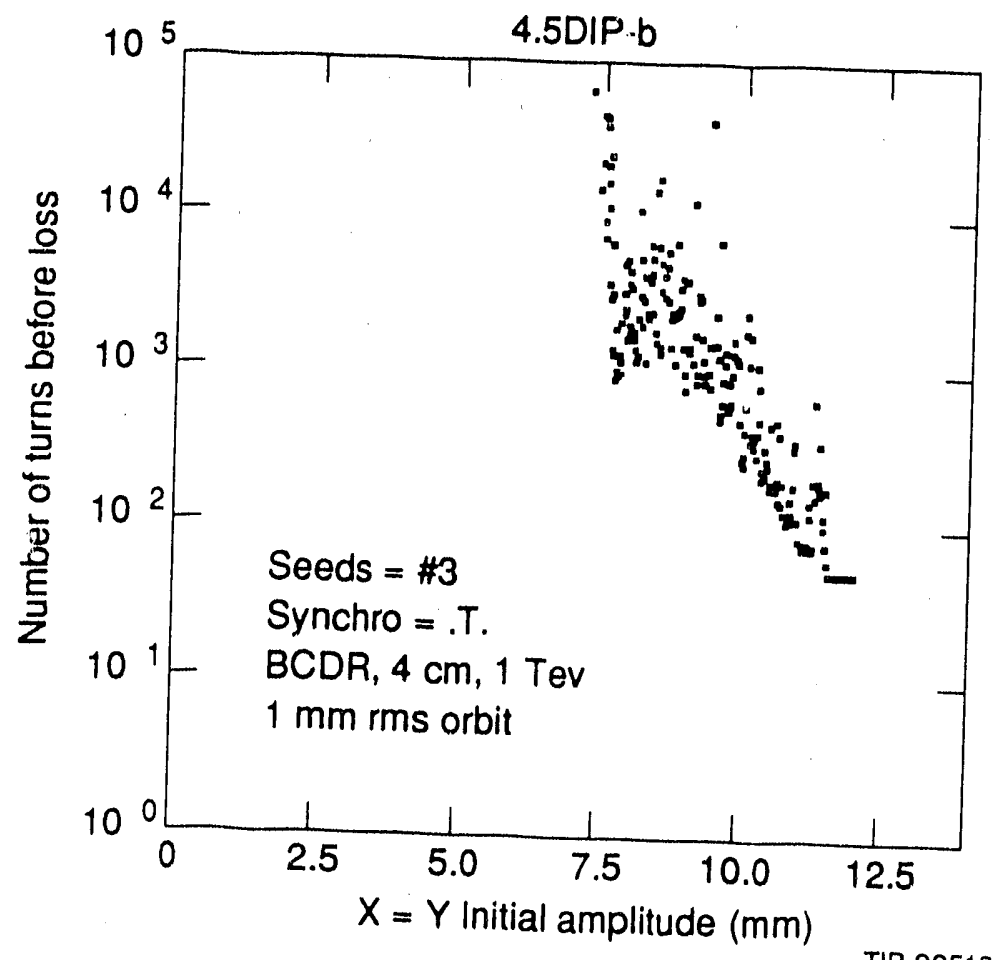

Figure 7: Number of turns survived in simulation of 1986 CDR Collider vs. initial betatron
oscillation amplitude. 


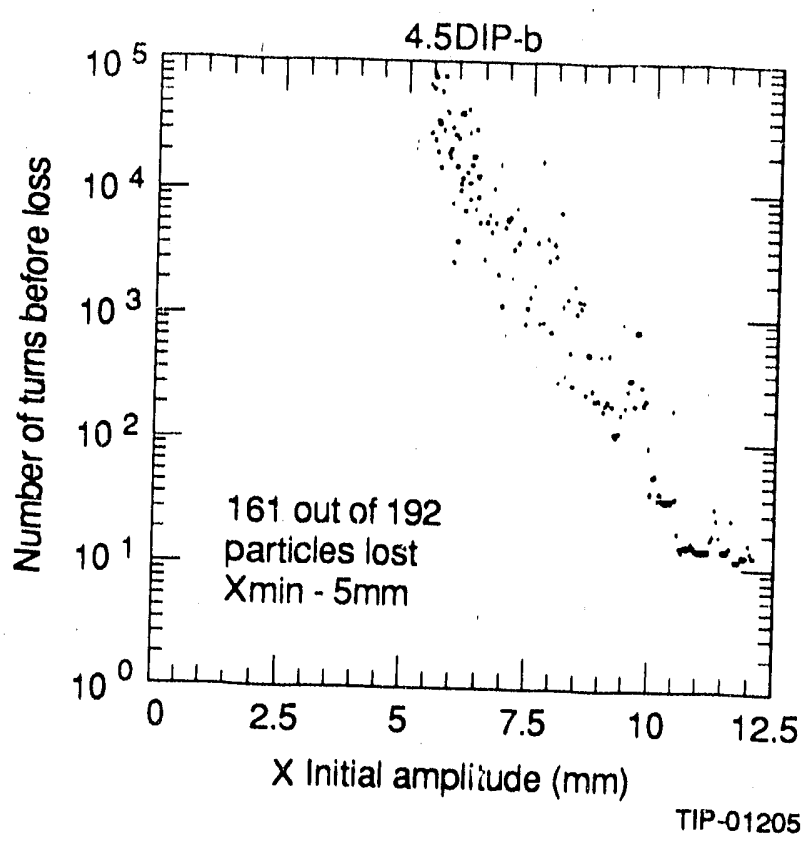

Figure 8: Number of turns survived in simulation of 1989 Collider design vs. initial betatron oscillation amplitude. Bore tube correctors are not used in this simulation.

\subsection{Persistent Currents}

Nonlinear fields arising from persistent currents have long been a serious concern in the SSC design effort [11]. The inclusion of bore tube correctors was motivated by these effects as was the emphasis on conductors with small filament size. One of the attractive features of separated function lattices is the elimination of the need for precise alignment of the bending magnets. If, however, the bending magnets contain sufficient nonlinearities, that advantage is lost unless one corrects the problem at its origin, hence, the bore tube correctors. The dipole moments due to persistent currents are directly proportional to the filament diameter, and it was recognized early on that a filament size of approximately $3 \mu \mathrm{m}$ would permit the use of lumped correctors at an injection energy of $1 \mathrm{TeV}$.

Operation of the Fermilab Tevatron collider revealed that "persistent" current multipoles in the superconducting magnets were not persistent at all but slowly drifted with time. With the Tevatron held at its injection energy, the chromaticity would change by 70 units over a period of a few hours [12]. Though these changes could be corrected using sextupole circuits, once the magnets began to ramp the chromaticity changed in some then unknown fashion often times leading to beam loss and emittance growth.

A series of magnet measurements were initiated at Fermilab to understand how the sextupole moment of the dipoles behaved and, hopefully, uncover the cause [13]. Figure 9 shows the sextupole coefficient $b_{2}$ as a function of excitation current. The arrows indicate increasing time and the data shown here are for a continuously ramping cycle. When the injection current is held constant for a period of time, $b_{2}$ drifts positively as shown in Figure 10 . 'The one unit drift in $b_{2}$ (corresponding to a change in the chromaticity of 30 units) took 15 minutes. As the magnet current began to change, $b_{2}$ almost immediately jumps down to rejoin the normal hysteresis curve. Once this behavior had been identified, the proper chromaticity 


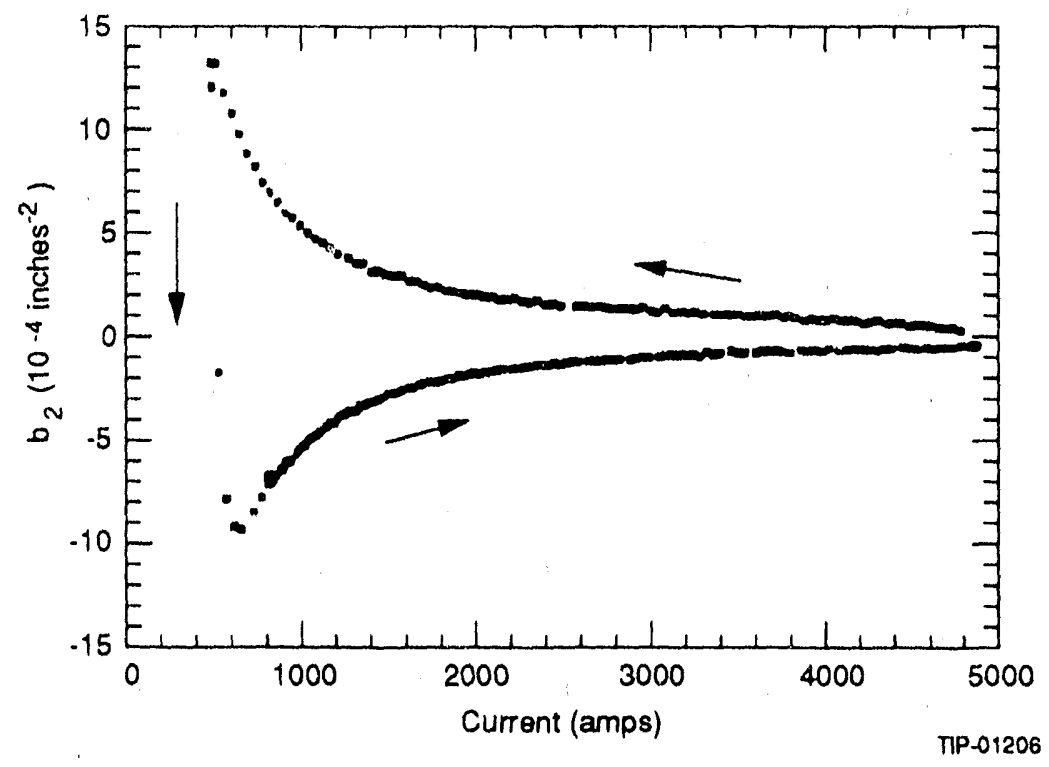

Figure 9: Sextupole moment in a model Tevatron dipole magnet as function of excitation current. The arrows indicate increasing time; the magnet was ramped continuously.

corrections could be implemented and the machine operation was greatly enhanced.

The cause of the drifting magnetic moments is thought to be the flux creep effect, which was first analyzed by Anderson in 1962 [14]. Flux creep varies logarithmically with time, a dependence seen in the magnet data. (See Figure 11.) The parameters of the superconducting cable which determine the creep rates are not understood. HERA magnets exhibit significantly different creep rates, dependent on the cable source, operating temperature, and the ramp history of the magnet [15].

\subsection{Design Approach}

The scale of the SSC presents a new frontier of reliability for particle accelerators. Successful though the first superconducting synchrotron, the Tevatron, has been, a simple extrapolation of its performance to the SSC would imply near zero availability for physics. Further, the design of the SSC mist be such as to provide avenues for flexibility and improvement beyond the initial goals established by the 1983 HEPAP subpanel [16]. These considerations coupled with the warning signals noted in both simulation and experiment persuaded the SSCL staff to increase the conservatism of the design. While it was clear from the outset of the design effort in 1984 that the SSC could not be as conservative, in a dynamical sense, as the Tevatron, a comparable dynamical simplicity was adopted as a goal. That is, it should be possible to initiate the experimental program at the SSC with only a short and methodical commissioning period with beam.

The three major parameters chosen for examination were ulie quadtupole spacing, the injection energy, and the coil diameter of the superconducting magnets. A decrease of the first and an increase of the other two are steps toward a more constrvative design. The 


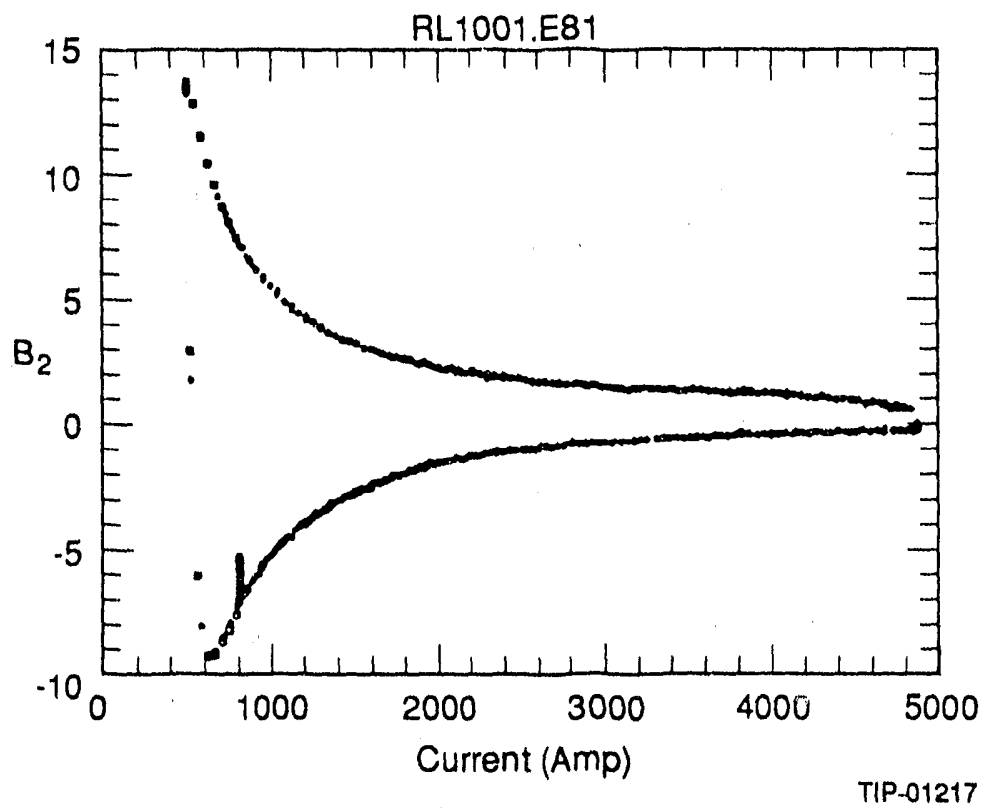

Figure 10: Sextupole moment in a model Tevatron dipole magnet as function of excitation current. Here, the current was held constant at $800 \mathrm{~A}$ for $15 \mathrm{~min}$.; the sextupole moment drifted by more than 2 units.

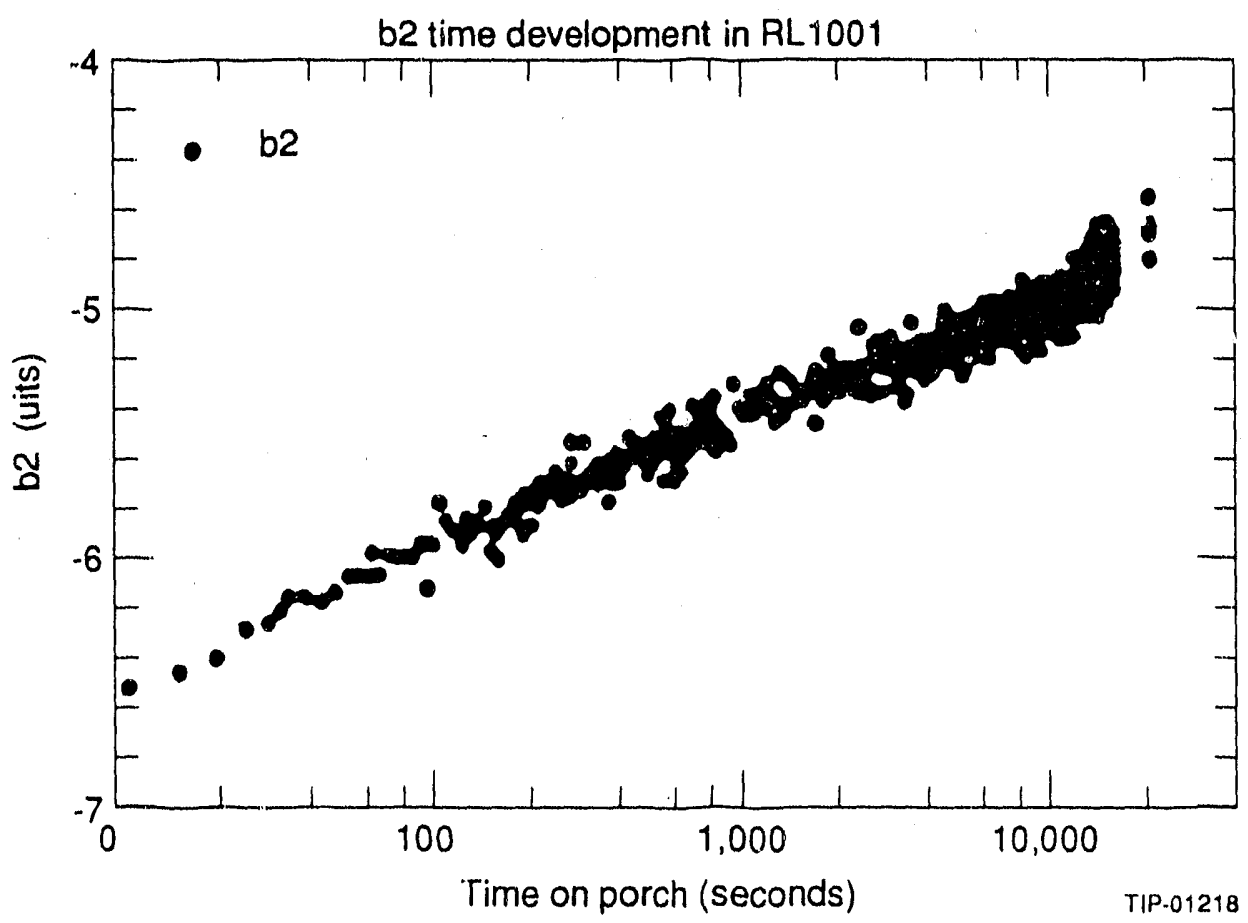

Figure 11: Sextupole moment of short Tevatron dipole magnet as function of time at the injection current. The data were taken after a standard accelerator ramp. The logarithmic dependence is indicative of flux creep. 
magnitudes of the changes inevitably involve judgement as will be clear from the discussion below.

As mentioned previously, a fourth parameter, the filament size of the superconducting cable, would also lead to smaller persistent current multipoles. High quality superconducting strand has been successfully produced in quantity with a filament diameter of $6 \mu \mathrm{m}$. It is entirely possible that a smaller filament size can be achieved. But, a development program would be required, and it has been decided not to rely on improvement in this parameter to enhance design conscrvatism.

\section{Recent Parameter Changes}

In the subsections to follow, we take up the three parameters in sequence. The basic performance specified for the $\mathrm{SSC}$ is the provision of $40 \mathrm{TeV}$ center-of-momentum collisions at a luminosity of $10^{33} \mathrm{~cm}^{-2} \mathrm{sec}^{-1}$. The nominal beam emittance is $\epsilon_{N}=\pi \sigma^{2} \% / \beta=1 \pi$ mm-mrad, and the momentum spread at injection is $\sigma_{p} / p=1 \times 10^{-4}$. But, it must be assumed that luminosity in excess of $10^{34} \mathrm{~cm}^{-2} \mathrm{sec}^{-1}$ will ultimately be desired and such higher performance may almost surely be accompanied by the need to accommodate larger emittance.

\subsection{Quadrupole Spacing}

The first order effect that the quadrupole spacing has on the accelerator performance is the determination of the beam size. For a quadrupoie spacing (half cell length) $L$ and a betatron phase advance $\mu$ per cell, the maximum amplitude function of the cell is given by

$$
\hat{\beta}=\frac{L}{\sin \mu / 2} \sqrt{\frac{1+\sin \mu / 2}{1-\sin \mu / 2}}
$$

which, for $90^{\circ}$ cells, reduces to

$$
\hat{\beta}=3.41 L \text {. }
$$

The dispersion function, which describes the closed orbit for off-momentum particles, varies as the square of the quadrupole spacing. Its maximum value within a cell is

$$
\hat{D}=\frac{L^{2}}{R \sin ^{2} \mu / 2} \cdot\left(1+\frac{1}{2} \sin \mu / 2\right)=2.71 \frac{L^{2}}{R}
$$

where $R$ is the average radius of the arc; the last expression is for $90^{\circ}$ cells.

The maximum rms beam size within a cell is then

$$
\hat{\sigma}=\sqrt{\frac{\hat{\beta} \epsilon_{N}}{\pi \gamma}+\hat{D}^{2}\left(\frac{\sigma_{p}}{p}\right)^{2}}
$$


where $\epsilon_{N}$ is as defined above, $\gamma$ is the ratio of beam energy to the proton rest energy, and $\sigma_{p} / p$ is the rms momentum spread of the beam.

In addition to the physical extent of the beam being smaller, a shorter half cell length can result in an increase in the linear aperture. As an example, we consider the one degree of freedom tine shift due to a normal multipole. If the transverse displacement $x$ and its conjugate variable are written in the form

$$
\begin{aligned}
x(s) & =a\left(\frac{\beta(s)}{\beta_{o}}\right)^{1 / 2} \cos \psi(s), \\
\left(\alpha x+\beta x^{\prime}\right) & =-a\left(\frac{\beta}{\beta_{o}}\right)^{1 / 2} \sin \psi,
\end{aligned}
$$

then the change in the particle's phase advance $\psi$ upon passing through a thin lens multipole is given by

$$
\Delta \psi=-\frac{\left(\beta \beta_{o}\right)^{1 / 2} \cos \psi \Delta x^{\prime}}{a}
$$

where

$$
\Delta x^{\prime}=-\frac{B_{o} b_{n}}{(B \rho)}\left[a\left(\frac{\beta}{\beta_{o}}\right)^{1 / 2} \cos \psi+D \delta\right]^{n} \Delta s .
$$

Here, $D$ is the dispersion function at the location of the error, $\delta$ is the mimentum deviation of the particle, $B_{0}$ is the ideal main guide field, $(B \rho)$ is the particle's mignetic rigidity, and $b_{n}$ is the multipole coefficient (defined on the next page).

The first-order tune shift due to a distribution of such multipoles is thus

$$
\Delta \nu=\left\langle b_{n}\left(\beta \beta_{o}\right)^{1 / 2} \cos \psi\left[a\left(\frac{\beta}{\beta_{o}}\right)^{1 / 2} \cos \psi+D \delta\right]^{n} / / a\right.
$$

where

$$
\langle f(s)\rangle \equiv \frac{1}{2 \pi} \frac{B_{o}}{(B \rho)} \int f(s) d s .
$$

The results for $n=0,1, \ldots, 5$ are given in Table 1 . In this table the particle emittance is defined as $\zeta \equiv a^{2} / \beta_{0}$.

The entries in Table 1 for $n=1$ and $n=2$ are the familiar results for the tune shift due to quadrupole errors and the chromaticity due to sextupole errors, respectively. Noting the above scaling of $\beta$ and $D$ with half-cell length $L$, the chromaticity due to systematic sextupole errors is seen to vary as $L^{3}$. The higher order multipoles have even higher dependences on half-cell length; for instance, the tune shift of off-momentum particles due to decapole errors $\left(b_{4}\right)$ varies as the quadrupole s! acing to the seventh power.

One would expect to be able to make a significant extension of the linear region of the aperture by shortening the half cell length. Additionally, the physical extent of the beam would be reduced. The half cell length chosen for the Collider rings is $90 \mathrm{~m}$. Compared to the $114.25 \mathrm{~m}$ lattice of September, 1987 , this amounts to approximately a $20 \%$ reduction in beam size and the chromaticity produced by systematic sextupole errors is cut in half. 


\begin{tabular}{|l|l|}
\hline $\mathbf{n}$ & First Order Tune Shift, $\Delta \nu$ \\
\hline \hline 0 & 0 \\
\hline 1 & $(1 / 2)\left\langle\widehat{\beta b_{1}}\right\rangle$ \\
\hline 2 & $\left\langle b_{2} \overline{\beta D\rangle \delta}\right.$ \\
\hline 3 & $(3 / 8)\left\langle b_{3} \beta^{2}\right\rangle \zeta+(3 / 2)\left\langle b_{3} \beta D^{2}\right\rangle \delta^{2}$ \\
\hline 4 & $(3 / 2)\left\langle b_{4} \beta^{2} D\right\rangle \zeta \delta+2\left\langle b_{4} \beta D^{3}\right\rangle \delta^{3}$ \\
\hline 5 & $(5 / 16)\left\langle b_{5} \beta^{3}\right\rangle \zeta^{2}+(15 / 4)\left\langle b_{5} \beta^{2} D^{2}\right\rangle \zeta \delta^{2}+(5 / 2)\left\langle b_{5} \beta D^{4}\right\rangle \delta^{4}$ \\
\hline 6 & $(15 / 8)\left\langle b_{6} \beta^{3} D\right\rangle \zeta^{2} \delta+(15 / 2)\left\langle b_{6} \beta^{2} D^{3}\right\rangle \zeta \delta^{3}+3\left\langle b_{6} \beta D^{5}\right\rangle \delta^{5}$ \\
\hline
\end{tabular}

Table 1: First order perturbation tune shifts $\Delta \nu$ due to multipoles of order $n$.

\subsection{Injection Inergy}

Due to the adiabatic damping of betatron oscillations as the energy of the beam is raised, a higher injection energy for the Collider rings impiles a smaller beam size and hence reduced aperture requirements. (Refer to Eq. 3.) More importantly, the "persistent" current multipcles at injection will be lessened. As the transport current in the superconducting magnets is raised the persistent currents are reduced and hence the values of the multipole coefficients due to them are reduced as well. This is evident in the curves of Figure 9 and
Figure 10.

The field error $\Delta B$ due to persistent currents in the superconducting cable can be expressed in a series expansion in the transverse coordinate $x$ as

$$
\Delta B=B_{\circ} \sum_{n} b_{n} x^{n}
$$

where the $b_{n}$ are the normal multipole coefficients. (This expression assumes up-down symmetry is preserved.) The dominant error field in the dipole magnets is the sextupole, or $b_{2}$ coefficient, which affects the chromaticity of the accelerator according to

$$
\Delta \xi=\left\langle b_{2} \beta D\right\rangle
$$

where the average is taken over the bending magnets. The chromaticity due to the $b_{2}$ in the dipoles for the CDR machine is 3400 . (The natural chromaticity of the CDR Collider is -134.) Since the total chromaticity needs to be adjusted to $\xi<5$ for the eventual operation of the SSC, this would entail strong sextupole correctors which must correct the chromaticity to an accuracy of $.15 \%$.

By doubling the injection energy from $1 \mathrm{TeV}$ to $2 \mathrm{TeV}$ the sextupole coefficient is cut by over a factor of two (see Fig. 12) and coupled with the cell length change discussed in the previous section the chromaticity of the Collider ring due to persistent current $b_{2}$ is reduced to 700 units. The reduction of $b_{2}$ iracreases the linear aperture as well. It also relaxes the alignment requirements of the dipoles enough to eliminate the need for bore tube correctors. In addition, the betatron oscillation amplitudes of the beam particles are reduced by $30 \%$ 
due to adiabatic damping and, in conjunction with the new half cell spacing, the beam size is reduced by $50 \%$.

\subsection{Magnet Coil Diameter}

The good field region of the Collider magnets can be increased by increasing the magnet coil diameter, keeping the field shape the same. Rewriting Eq. 4,

$$
\frac{\Delta B(x)}{B_{o}}=\sum_{n} R_{o}^{n} b_{n}\left(\frac{x}{R_{o}}\right)^{n}
$$

we can see that if the transverse dimensions of the magnet (indicated by the coil radius $R_{0}$ ) are increased while keeping the left-hand side a fixed function of $x / R_{o}$, then

$$
b_{n} \sim \frac{1}{R_{o}^{n}}
$$

This scaling law applies to the systematic, or average, multipoles in the magnets which are generated by the magnet geometry or by persistent currents.

We consider also the random coil placement errors in an ensemble of magnets. The variance of a distribution of multipoles will depend upon the coil radius according to

$$
\sigma_{b_{n}}^{2} \sim \frac{1}{N} \sum_{i}\left(\frac{1}{R_{i}^{n}}-\frac{1}{R_{o}^{n}}\right)^{2}
$$

which, after writing $R_{i}=R_{o}+\Delta R_{i}$, yields

$$
\sigma_{b_{n}} \sim \frac{1}{R_{o}^{n+1}} \Delta R_{r m s} .
$$

If coil placement errors are independent of the scale of the magnet, then $\sigma_{b_{n}} \sim 1 / R_{o}^{n+1}$. On the other hand, if placement errors scale in proportion to the size of the magnet, then $\sigma_{b_{n}} \sim 1 / R_{0}^{n}$. In either case higher order multipoles are significantly reduced by increasing the coil radius. For comparison studies, the geometric mean of these two cases was used to perform the scaling of randorn multipoles, i.e.,

$$
\sigma_{b_{n}} \sim \frac{1}{R_{o}^{n+1 / 2}}
$$

It should be noted that due to the random errors in coil placement, there will be a statistical average multipole whose rms width is given by

$$
\sigma_{\left\langle b_{n}\right\rangle}=\frac{\sigma_{b_{n}}}{\sqrt{N}}
$$

where $N$ is the number of magnets in a Collider ring. Since $N=4800$ these average multipoles are insignificant when compared to the systematic multipoles due to the magnet geometry. 


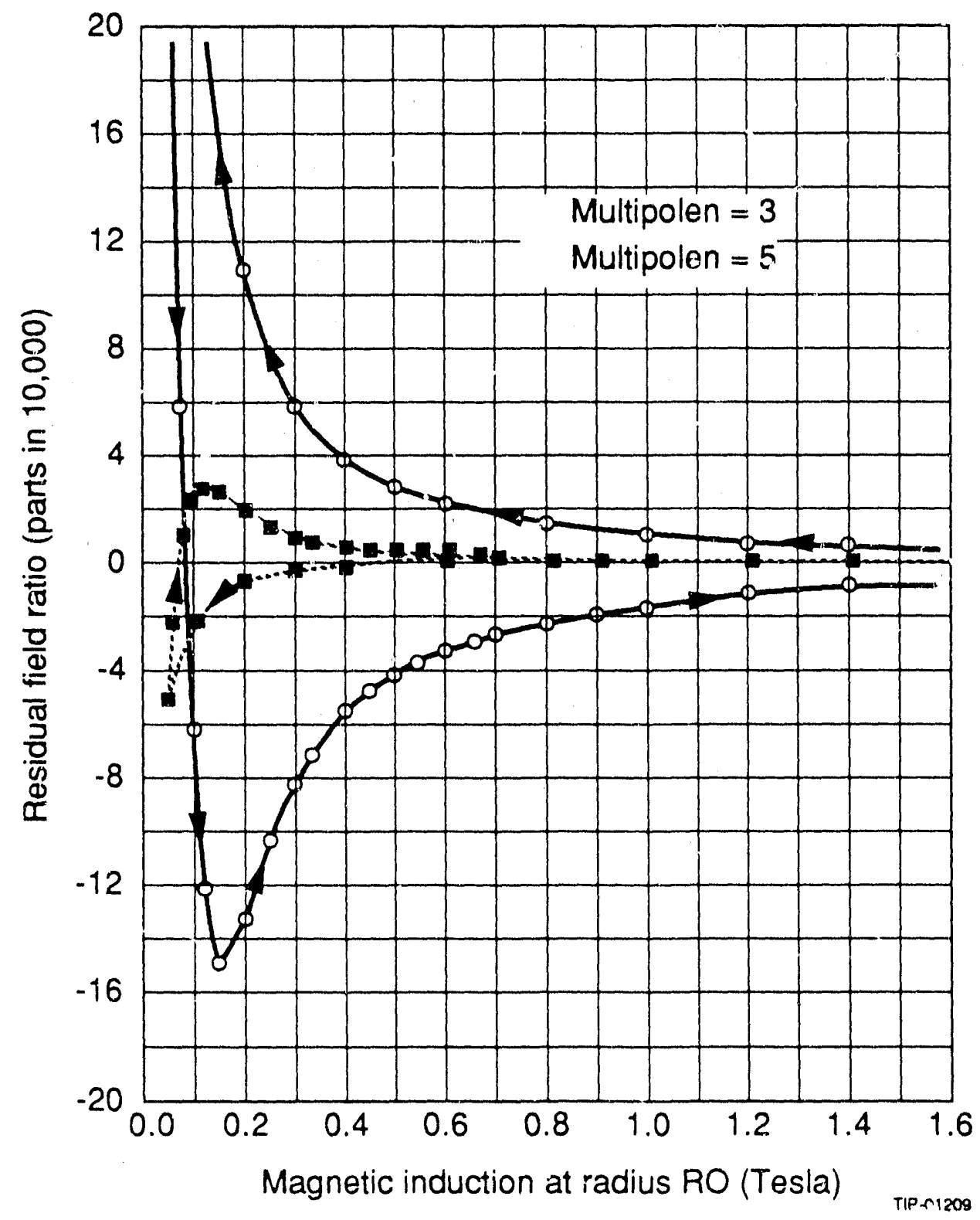

Figure 12: Calculated sextupole moment (circles) and decapole moment (squares) for $4 \mathrm{~cm}$ Collider dipole magnet as function of central field. The injection field is $0.33 \mathrm{~T}$ for $1 \mathrm{TeV}$ injection, $0.66 \mathrm{~T}$ for $2 \mathrm{TeV}$ injection. 


\begin{tabular}{|c|c|c||c|c|}
\hline Multipole & systematic & random & HERA & Tevatron \\
\hline$b_{1}$ & 0.00 & 0.70 & 0.68 & 0.57 \\
\hline$a_{1}$ & 0.00 & 0.70 & 1.74 & 0.80 \\
\hline$b_{2}$ & 0.50 & $2.01^{*}$ & 2.00 & 2.80 \\
\hline$a_{2}$ & 0.05 & 0.62 & 0.35 & 0.96 \\
\hline$b_{3}$ & 0.05 & 0.34 & 0.16 & 0.49 \\
\hline$a_{3}$ & 0.05 & 0.69 & 0.52 & 0.16 \\
\hline$b_{4}$ & 0.05 & 0.59 & 0.36 & 0.58 \\
\hline$a_{4}$ & 0.05 & 0.14 & 0.11 & 0.23 \\
\hline$b_{5}$ & 0.05 & 0.06 & 0.04 & n.a. \\
\hline$a_{5}$ & 0.05 & 0.16 & 0.07 & n.a. \\
\hline$b_{6}$ & -0.07 & 0.075 & 0.07 & 0.22 \\
\hline$a_{6}$ & 0.05 & 0.034 & 0.03 & n.a. \\
\hline
\end{tabular}

* sorted to 0.40

Table 2: Systematic and random multipole values used in the simulations of SSC Collider rings at injection $(2 \mathrm{TeV})$. The "units" used are $10^{-4} / \mathrm{cm}^{n}$. The two right-hand columns show HERA and Tevatron dipole magnet random multipoles (measured and scaled with coil
diameter) for comparison.

Simulations were initiated in late 1989 to compare the dynamic apertures of dipole magnets with $4 \mathrm{~cm}$ and $5 \mathrm{~cm}$ inner coil diameters. In an effort to speed up the analysis and to better approach the $10^{6}-10^{7}$ turn regime, a new code was developed (see [17]) in which the nonlinear kicks delivered from the dipole magnets were grouped into four regions per cell (at each major quadrupole and at the midpoints between quadrupoles) with appropriate weighting. Table 2 shows the $4 \mathrm{~cm}$ dipole systematic and random multipoles (both normal, $b_{n}$, and skew,$\left.a_{n}\right)$ used in these simulations of the SSC Collider rings at injection. A discussion on the choice of values can be found in [18]. The values for $5 \mathrm{~cm}$ magnets used in the simulations were obtained using the scaling laws described above.

Figure 13 shows the survival plot for the $4 \mathrm{~cm}$ dipole simulation using the error distribution of Table 2. The solid line is drawn for comparison with the $5 \mathrm{~cm}$ simulation, which is shown in Figure 14. The gain in dynamic aperture in going from $4 \mathrm{~cm}$ to $5 \mathrm{~cm}$ inner coil diameter is approximately a factor of 1.6 (which, coincidentally, is approximately $(5 / 4)^{2}$ ). These results, and other presentations, were reviewed by the SSC Machine Advisory Committee in December, 1989. The committee's conclusions strongly recommended a change in magnet coil diameter from $4 \mathrm{~cm}$ to $5 \mathrm{~cm}$, a program SSCL has since adopted. 


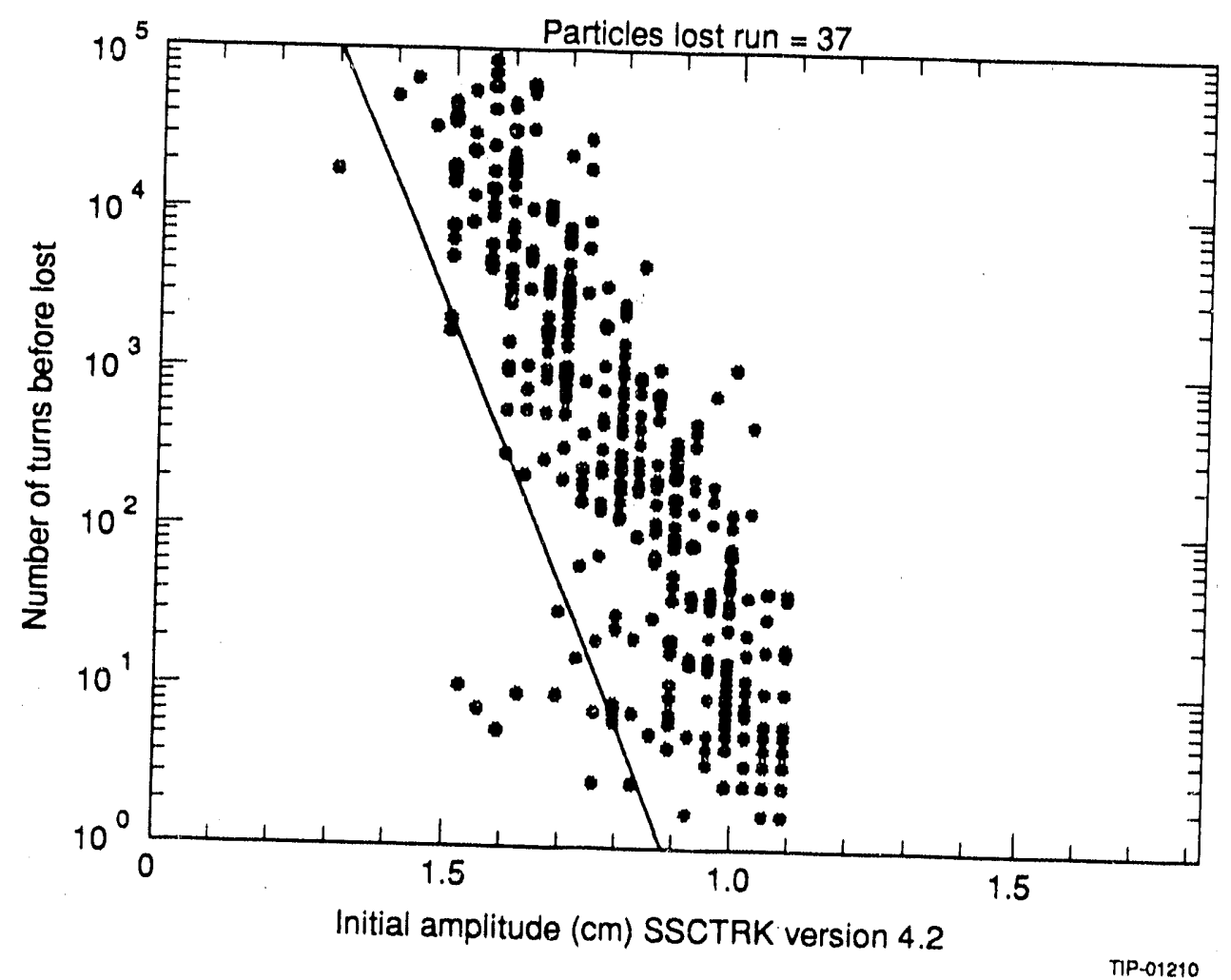

Figure 13: Survival plot for machine with $4 \mathrm{~cm}$ dipole magnet inner coil diameter.

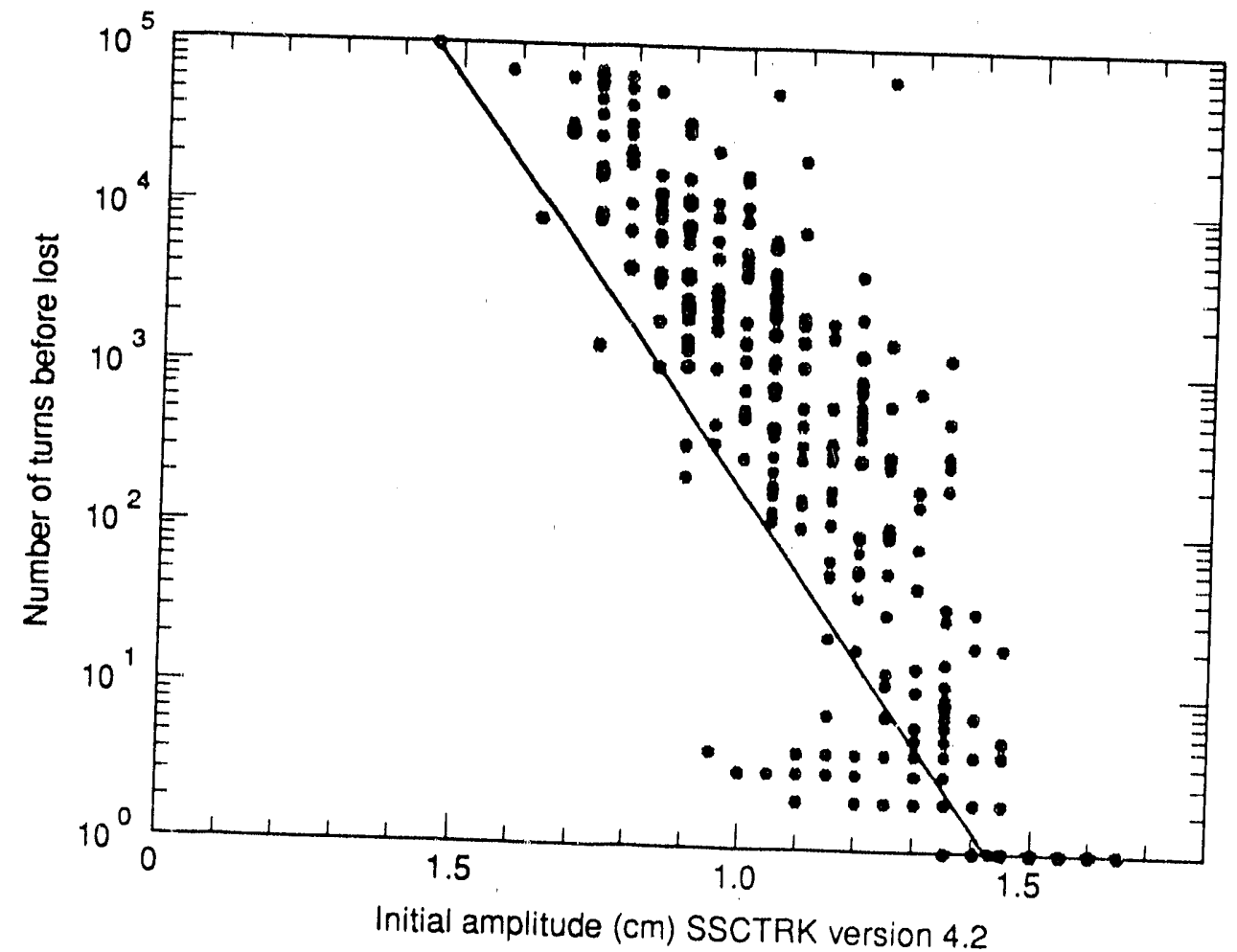

Figure 14: Survival, lot for $5 \mathrm{~cm}$ machine. 


\section{Summary and Conclusions}

The purpose of this paper was to describe a process with duration over a year in which three basic parameters of the SSC were subjected to scrutiny and, as a result of that scrutiny, modifications were recommended in them. The recommended modifications were in the direction of increase in design conservatism.

The recent changes of the parameters were made in an effort to improve the Collider, but obviously come with a price. Firstly, the quadrupole spacing has been decreased from $114.25 \mathrm{~m}$ to $90 \mathrm{~m}$; this parameter change reduces the beam size slightly, but in addition increases the linear aperture. It also implies more quadrupole magnets (of longer lengths) for the Collider rings which take up space that could have been used for bending. (This change does not significantly affect the maximum energy of the Collider.) Secondly, the injection energy of the Collider has been raised from $1 \mathrm{TeV}$ to $2 \mathrm{TeV}$, reducing the major source of nonlinearity in the synchrotrons at the most critical energy. The major consequence of this change is an increase in the size of the High Energy Booster. And thirdly, the inner coil diameter of the main dipole magnets has been increased from $4 \mathrm{~cm}$ to $5 \mathrm{~cm}$ with a proportionately larger magnet cross section. This change increases the good field region of the dipole magnet, but obviously has a major impact on its cost.

Table 3 summarizes the parameter changes and their effects on beam size and chromaticity. The last two rows compare the beam size and the chromaticity due to the persistent current sextupole moment relative to the September, 1987, (ISP) design.

With the new parameters, the required beam size for the design emittance and momentum spread is cut by $40 \%$ and the chromaticity at injection is reduced by almost $90 \%$. The increased injection field eliminates the need for bore tube correctors from the main bending magnets simplifying their design, construction, installation, and operation. Construction simplification for an accelerator of this scale was considered an important design consideration.

'The increase in the coil diameter of the main bending magnets was the most controversial of the changes, and one that had been under discussion since the Reference Designs Studies of 1984. The coil diameter was a matter of considerable discussion at the DOE review of the CDR in April of 1986 as noted in the preceding text. At its meeting of December 1989, the SSC Machine Advisory Committee recommended that the step to the $5 \mathrm{~cm}$ coil diameter must be undertaken, and that recommendation was accepted by the SSCL Director. With this change, the dynamic aperture for particles circulating at the injection energy for $10^{5}$ turns is increased by over $50 \%$. In addition, the scaling of the magnet size has the result, that less current density is required to produce the same central field. A safety margin of $9 \%$ in the quench current of the dipole magnets will result; the safety margin for the $4 \mathrm{~cm}$ design had been calculated to be $4 \%$.

It is intended that the parameter changes outlined in this report significantly increase the reliability and availability of the SSC. The course of commissioning will surely be more predictable, hence shorter. Downtirne due to quenches, component failure, and repairs is inevitable; the changes seek to lessen additional time demands for accelerator studies. Fur- 


\begin{tabular}{|l|cc|ccc|c|c|}
\hline & CDR & ISP & $90 \mathrm{~m}$ & $2 \mathrm{TeV}$ & $5 \mathrm{~cm}$ & SCDR & \\
\hline$\epsilon_{N}$ & 1 & 1 & 1 & 1 & 1 & 1 & $\pi \mathrm{mm}-\mathrm{mr}$ \\
$\sigma_{p} / p$ & 0.175 & 0.175 & 0.175 & 0.175 & 0.175 & $0.096(0.175)$ & $\times 10^{-3}$ \\
$\hat{\beta}$ & & & & & & & \\
$\hat{D}$ & 332 & 388 & 305 & 388 & 388 & 305 & $\mathrm{~m}$ \\
& 3.92 & 3.05 & 1.82 & 3.05 & 3.05 & 1.82 & $\mathrm{~m}$ \\
$\gamma_{\text {inj }}$ & 1066 & 1066 & 1066 & 2132 & 1066 & 2132 & \\
$\left|\left\langle b_{2}\right\rangle\right|$ & 7 & 7 & 7 & 3 & 4.48 & 1.92 & $\mathrm{~m}^{-2}$ \\
\hline & & & & & & & \\
$\sigma_{\beta}$ & 0.56 & 0.60 & 0.53 & 0.43 & 0.60 & 0.38 & $\mathrm{~mm}^{-2}$ \\
$\sigma_{D}$ & 0.69 & 0.53 & 0.32 & 0.53 & 0.53 & $0.17(0.32)$ & $\mathrm{mm}$ \\
$\sigma_{D} / \sigma_{\beta}$ & 1.23 & 0.88 & 0.60 & 1.25 & 0.88 & $0.46(0.84)$ & \\
& & & & & & & \\
$\sigma_{T \text { TOT }}$ & 0.88 & 0.81 & 0.62 & 0.68 & 0.81 & $0.42(0.49)$ & $\mathrm{mm}$ \\
Rel. $\sigma$ & 1.10 & 1.00 & 0.77 & 0.85 & 1.00 & $0.52(0.61)$ & \\
Rel. $\xi$ & 1.10 & 1.00 & 0.47 & 0.43 & 0.64 & 0.13 & \\
\hline
\end{tabular}

CDR $=1986$ SSC Conceptual Design Report lattice and parameters; ISP $=1987$ Invitation for Site Proposal lattice and parameters; SCDR $=1989$ Site-specific Conceptual Design Report lattice and parameters; $\sigma_{\beta}=\sqrt{\epsilon_{N} \beta / \pi \gamma}, \sigma_{D}=D\left(\sigma_{p} / p\right)$, and $\sigma_{T O T}=\sqrt{\sigma_{\beta}^{2}+\sigma_{D}^{2}}$.

Table 3: Comparison of beam sizes and chromaticities due to the various parameter changes. The SCDR design calls for an rms momentum spread at injection of $10^{-4}$. The numbers in parentheses correspond to the momentum spread of the CDR design. 
ther, the more conservative design readily allows for the possibility of accelerator upgrades to meet the ever increasing demands of the experimental program, therefore extending the useful life of the laboratory.

Acknowledgements. The authors would like to thank H. Edwards, R. Steining, and A. Chao for useful information, discussions, and comments during the preparation of this document.

\section{References}

[1] D. A. Edwards and M. J. Syphers, "Experimental Particle Tracking," Frontiers of Particle Beams; Observation, Diagnosis and Correction, Lecture Notes in Physics 343, Springer-Verlag, 1989.

[2] A. W. Chao et al., Phys. Rev. Lett. 61, 2752(1988).

[3] D. A. Edwards, "Aperture Task Force Interim Report -.. Aperture Criterion Group," SSC note SSC-22, 1985.

[4] See A. Hilaire, "Dynamical Aperture at the SpS," Proc. of Second Advanced ICFA Beam Dynamics Workshop, CERN 88-04, Lugano, 1988, and L. Evans, et al., "The Nonlinear Dynamic Aperture Experiment in the SpS," EPAC Conference, Rome, 1988.

[5] J. Gareyte, et al., "Effect of Sextupoles on the Single Particle Dynamics in the CERN SpS," CERN SPS/88-45, 1988.

[6] R. Gerig, "Main Ring Dynamic Aperture Studies," FNAL internal report EXP-164, May, 1989.

[7] M. Syphers, "Beam-Gas Scattering Lifetimes in the Fermilab Main Ring," FN-484, Fermilab, 1988.

[8] L. Evans, et al., "Non Linear Dynamic Aperture Experiments," SpS Improvement Report No. 209, CERN internal report, 1987.

[9] R. E. Gerig, L. Michelotti, Y. Chao, "Modeling Nonlinear Behavior in the Fermilab Main Ring," Proc. of 1989 IEEE Part. Accel. Conf., IEEE 89CH2669-0.

[10] L. Schachinger and Y. Yan, "Recent SSC Dynamic Aperture Measurements from Simulations," SSC note SSC-N-664, 1989.

[11] "Superconducting Super Collider, Reference Designs Study for the U.S. Department of Energy," May 8, 1984.

[12] D. A. Finley, et al., "Time Dependent Chromaticity Changes in the Tevatron," Proc. of 1987 IELE Part. Accel. Conf., IEEE 87CH2387-9. 
[13] D. A. Herrup, é al., "Time Variations of Fields in Superconducting Magnets and their Effects on Accelerators," presented at 1988 Applied Superconductivity Conference, San Francisco, CA, August, 1988 (also, Fermilab note TM-1543).

[14] P. W. Anderson, "Theory of Flux Creep in Hard Superconductors," Phys. Rev. Lett. 9, 309(1962).

[15] H. Bruck, et al., "Performance of the Superconducting Magnets for the HERA Accelerator," 14th International Conference on High Energy Accelerators, Tsukuba, Japan, 1989.

[16] "Report of the 1983 HEPAP Subpanal on New Facilities for the U.S. High Energy Program," U.S. Department of Energy, Office of Energy Research, Division of High Energy Physics, DOE/ER-0169, July 1983.

[17] D. Ritson, "SSC'TRK: A Particle Tracking Code for the SSC," SSC note (to be released).

[18] T. Garavagiia, K. Kaufmann, and R. Stiening, "Application of the SSCTRK Numerical Simulation Program to the Evaluation of the SSC Magnet Aperture," SSC note SSCL265. 

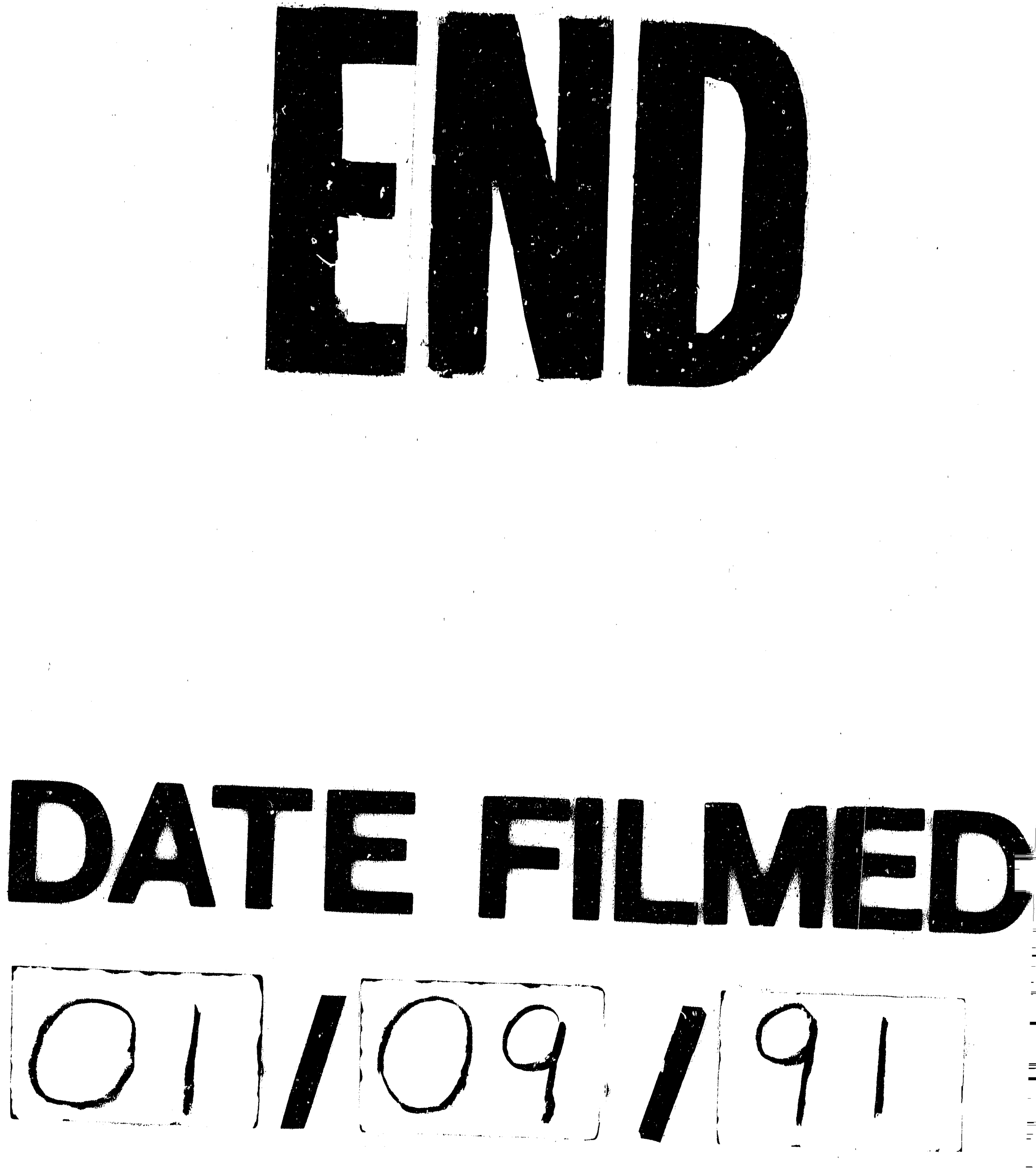
